\title{
Overview of knowledge flow in a software development process
}

\begin{abstract}
Knowledge flow (KF) and workflow have many definitions in the literature. The development of information technology has led many organizations to become more focused on knowledge than on labor because KF is considered complementary to the software development process when used in the software development domain. Knowledge is the most valuable property about any business organization or academic institution; in addition the knowledge flow plays a part in elevating the competitiveness of teams. This paper presents the origin of KF research and its different definitions. It provides an overview of $\mathrm{KF}$ and workflow and the interaction mechanism between the two. A systematic review of the subject is also given.
\end{abstract}

Keyword: Knowledge flow; Workflow; Software development process 